\title{
TITLE:
}

\section{Critical exponents of Nikolaevskii turbulence}

\author{
$\operatorname{AUTHOR}(\mathrm{S}):$ \\ Tanaka, D
}

\section{CITATION:}

Tanaka, D. Critical exponents of Nikolaevskii turbulence. Physical Review E 2005, 71(2): 025203.

ISSUE DATE:

2005-02

URL:

http://hdl.handle.net/2433/49993

\section{RIGHT:}

Copyright 2005 American Physical Society 
PHYSICAL REVIEW E 71, 025203(R) (2005)

\title{
Critical exponents of Nikolaevskii turbulence
}

\author{
Dan Tanaka* \\ Department of Physics, Graduate School of Sciences, Kyoto University, Kyoto 606-8502, Japan
}

(Received 3 December 2004; published 17 February 2005)

\begin{abstract}
We study the spatial power spectra of Nikolaevskii turbulence in one-dimensional space. First, we show that the energy distribution in wave-number space is extensive in nature. Then, we demonstrate that, when varying a particular parameter, the spectrum becomes qualitatively indistinguishable from that of KuramotoSivashinsky turbulence. Next, we derive the critical exponents of turbulent fluctuations. Finally, we argue that in some previous studies, parameter values for which this type of turbulence does not appear were mistakenly considered, and we resolve inconsistencies obtained in previous studies.
\end{abstract}

DOI: 10.1103/PhysRevE.71.025203

PACS number(s): 05.45. $-\mathrm{a}, 47.52 .+\mathrm{j}, 47.54 .+\mathrm{r}, 82.40 .-\mathrm{g}$

The spontaneous formation of spatially periodic structure in reaction-diffusion systems was predicted by Turing in 1952 [1] and experimentally confirmed many years later $[2,3]$. The so-called Turing mechanism is now widely accepted, and we can retrieve many papers by searching for the keyword "Turing pattern," including a large number written this century [4]. Recently, we found evidence that the Turing instability in oscillatory systems can also cause an initially uniform state to evolve into a state characterized by spatiotemporal chaos instead of spatially periodic structure $[5,6]$. This type of chemical turbulence is exhibited by the equation

$$
\partial_{t} \psi(x, t)=-\partial_{x}^{2}\left[\epsilon-\left(1+\partial_{x}^{2}\right)^{2}\right] \psi-\left(\partial_{x} \psi\right)^{2},
$$

which was derived from a class of oscillatory reactiondiffusion systems by means of a phase reduction technique [6]. An equivalent equation was proposed by Nikolaevskii as a model of seismic phenomena [7]. The uniform steady state of Eq. (1), $\psi=0$, is unstable with respect to finite-wavelength perturbations when the small parameter $\epsilon$ is positive. However, this instability does not lead to spatially periodic steady states, because the equation possesses a Goldstone mode, due to its invariance under transformations of the form $\psi$ $\rightarrow \psi+$ const, and the corresponding marginally stable longwavelength modes interact with the unstable shortwavelength modes. As a consequence, spatially periodic steady states do not appear, and instead spatiotemporal chaos is realized supercritically $[8,9]$. Spatiotemporal chaos exhibiting a similar onset has been observed experimentally in electrohydrodynamic convection ("soft-mode turbulence") in homeotropically aligned nematic liquid crystals [10] and numerically in Rayleigh-Bénard convection under free-free boundary conditions [11]. In particular, Eq. (1) has been applied to the study of the former type of convective system. It is thus seen that this class of spatiotemporal chaos appears in many types of physical systems, and for this reason, studying Eq. (1) is important. In this paper, we study the statistical properties of the spatiotemporal chaos exhibited by Eq. (1) in one-dimensional space with periodic boundary conditions.

*Electronic address: dan@ton.scphys.kyoto-u.ac.jp
Also, we argue that in some previous works on Nikolaevskii turbulence, values of $\epsilon$ that are in fact inappropriate for studying this type of turbulence were used.

Equation (1) has two parameters, the bifurcation parameter $\epsilon$ and the system size $L$. First, we derive the $L$ dependence of the spatial power spectrum $S(q) \equiv\left\langle\left|v_{q}\right|^{2}\right\rangle$, where $v_{q}$ is the spatial Fourier transform of $v \equiv 2 \partial_{x} \psi$ and \langle\rangle represents a long-time average. The quantity $S(q) / L$ is plotted as a function of the wave number $q$ for $L=2^{9}, 2^{10}, 2^{11}$, and $2^{12}$ with $\epsilon=0.02$ in Fig. 1. There it is seen that $S(q) / L$ possesses a universal form independent of $L$. This implies that the energy distribution in wave-number space is an extensive quantity. In Ref. [12], the Lyapunov dimension and the Kolmogorov-Sinai entropy are studied for Eq. (1) in the cases $\epsilon=0.2,0.5$, and it is shown that these too are extensive quantities. However, these values of $\epsilon$ are too large for Eq. (1) to exhibit the type of spatiotemporal chaos in which we are interested, as we show below.

Second, we consider the $\epsilon$ dependence of the spatial power density spectrum $S(q) / L$. In the following, we consider only the single system size $L=2^{9}$, because, as mentioned above, $S(q) / L$ is independent of $L$ when $L$ is sufficiently large. The peaks of the spectrum broaden and merge

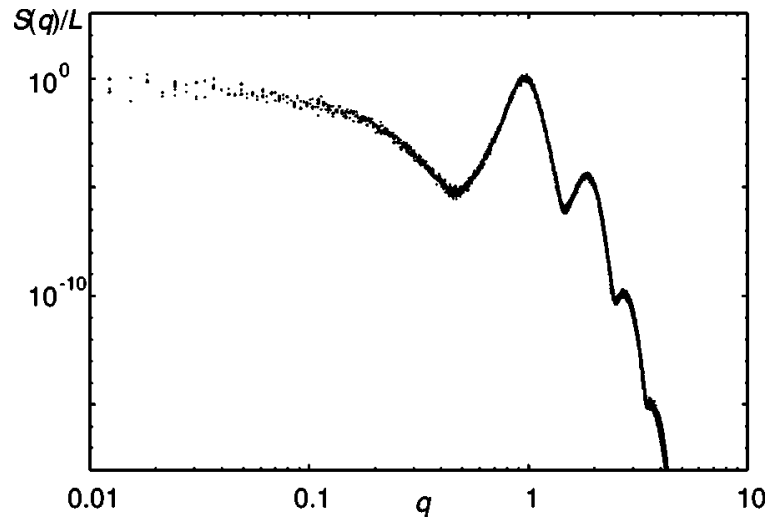

FIG. 1. Spatial power density spectrum $S(q) / L$ as a function of the wave number $q$ for $L=2^{9}, 2^{10}, 2^{11}$, and $2^{12}$ with $\epsilon=0.02$. The fact that these plots fall on a universal curve independent of $L$ implies the extensive nature of the energy distribution in wavenumber space. 


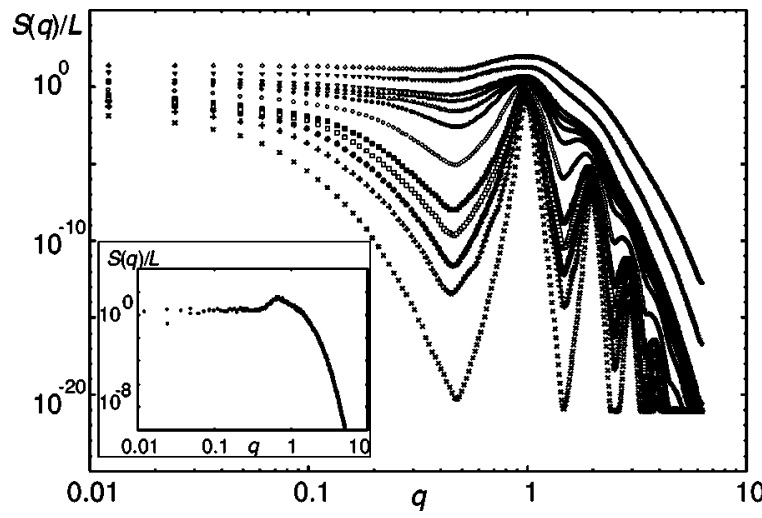

FIG. 2. Spatial power density spectrum $S(q) / L$ as a function of the wave number $q$ for several values of $\epsilon$ with $L=2^{9}$. From top to bottom, we have $\epsilon=0.4,0.2,0.1,0.08,0.06,0.04,0.02,0.01,0.008$, $0.006,0.004$, and 0.002 . The inset displays the spatial power density spectrum obtained from the well-known Kuramoto-Sivashinsky equation for the sake of comparison.

when $\epsilon$ increases, as shown in Fig. 2. In particular, when $\epsilon$ is larger than about 0.1 , the spectrum is qualitatively indistinguishable from that of the Kuramoto-Sivashinsky (KS) equation, $\partial_{t} \psi(x, t)=-\partial_{x}^{2}\left(1+\partial_{x}^{2}\right) \psi-\left(\partial_{x} \psi\right)^{2}$ [13]. This can be understood as follows. The spatiotemporal chaos exhibited by Eq. (1) with a sufficiently small $\epsilon$ arises from the uniform steady state $\psi=0$, owing to the interaction between the weakly stable long-wavelength modes and the unstable shortwavelength modes. The band of unstable modes has a width in wave-number space of order $\epsilon^{1 / 2}$, lying on either side of $q=1$. Therefore, unless $\epsilon^{1 / 2} \ll 1$, the weakly stable and unstable bands of modes cannot be distinguished, and thus the situation is effectively the same as that for the KS equation, in which case chaos arises through interactions among unstable long-wavelength modes. As a condition to ensure that the unstable and weakly stable bands of Eq. (1) are sufficiently separated, we conjecture that $\epsilon^{1 / 2}$ must be at least one order of magnitude smaller than 1 . Hence, in order to clearly observe the characteristic Nikolaevskii chaos exhibited by this equation, we believe that the $O(\epsilon) \leqslant 0.01$ is necessary. This leads us to conclude that the value $\epsilon=0.2$ and $\epsilon=0.5$ used in Refs. [12,14] (which employs a wavelet decomposition) are too large to observe this type of spatiotemporal chaos and that the power spectrum found in those works is actually that of KS spatiotemporal chaos. In fact, it is shown below that the exponents of the $\epsilon$ scaling for the chaotic fluctuations of Eq. (1) do not converge for $O(\epsilon) \geqslant 0.1$.

Noting that the spatiotemporal chaos exhibited by Eq. (1) results from the interaction between the long-wavelength modes near $q=0$ and the short-wavelength modes in the unstable band surrounding $q=1$, Matthews and Cox derived closed-form amplitude equations by hypothesizing that the behavior of the system can be described in terms of a quantity $v$ taking the form

$$
v=\epsilon^{3 / 4} A(X, T) e^{i x}+\text { c . c . }+\epsilon f(X, T),
$$

where $A$ and $f$ represent the slowly varying amplitudes of the two sets of modes, and we define $X \equiv \epsilon^{1 / 2} x$ and $T \equiv \epsilon^{1} t[15]$.

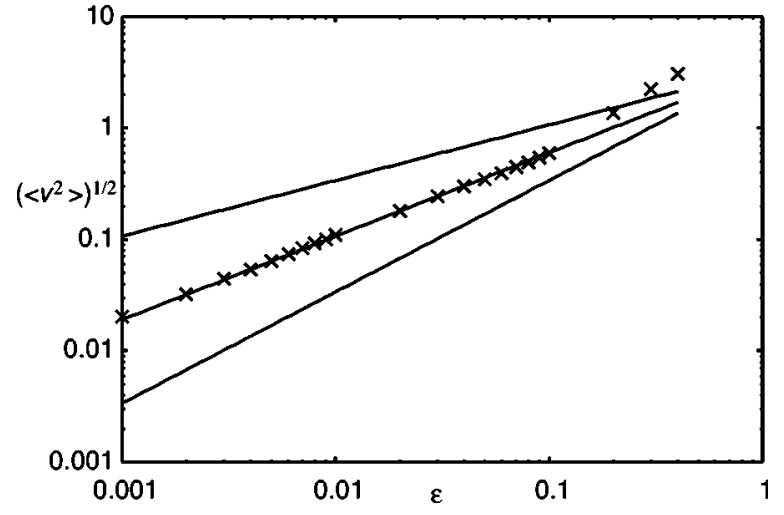

FIG. 3. $\epsilon$ dependence of $\sqrt{\left\langle v^{2}\right\rangle}$. The three lines, included for reference, have slopes of $2 / 4,3 / 4$, and $4 / 4$.

Also, Fujisaka et al. derived amplitude equations applicable in higher-dimensional spaces using the form Eq. (2) [16]. The validity of this form is supported by numerical results that show $\sqrt{\left\langle v^{2}\right\rangle} \propto \epsilon^{3 / 4}$ for $\epsilon \in[0.01,0.1][15]$. However, based on an analysis of the time series of the spatial Fourier amplitude of turbulent fluctuations for Eq. (1) with the fixed value $\epsilon=0.0001$, Tribelsky and Tsuboi conjectured the scaling $\sqrt{\left\langle v^{2}\right\rangle} \propto \epsilon^{1 / 2}$ in Ref. [9]. Also, in Ref. [17] it is shown that $\sqrt{\left\langle v^{2}\right\rangle} \propto \epsilon^{1}$ for $\epsilon \in[0.1,1]$. The discrepancy in the latter case seems to be easily accounted for, as it would appear that the result for the exponent reported in Ref. [17] had not yet converged, because the value of $\epsilon$ used there is too large. However, the situation is not so clear with regard to the apparent inconsistency reported in Ref. [9], because the value of $\epsilon$ used there is certainly sufficiently small. Furthermore, we believe that the numerical results of Ref. [15] are insufficient to establish the validity of the form given in Eq. (2) for the following reasons: (1,) The results were obtained for values of $\epsilon$ in a range of only one order, $\epsilon \in[0.01,0.1]$. It is quite likely that this small range is insufficient to yield a clear result for the power-law exponent. (2,) Studying only the order parameter $\sqrt{\left\langle v^{2}\right\rangle}$, we are able to examine the validity of only the assumed exponent $3 / 4$ for the amplitude of $e^{i x}$. Verifying the validity of the other exponents requires a different approach. (3,) Employing the spatial coordinate $X$ $=\epsilon^{1 / 2} x$ implies the assumption that the spatial scale of turbulent fluctuations is very much larger than that of the fundamental wave $e^{i x}$. This scale separation is ensured when $\epsilon^{1 / 2}$ $\ll 1$. Taking this condition as implying that $\epsilon^{1 / 2}$ can be no greater than 0.1 , we obtain the requirement $O(\epsilon) \leqslant 0.01$ to guarantee sufficient separation of scales. Now, to resolve the inconsistency among the results of the previous studies and to examine the validity of Eq. (2), we define some new order parameters and examine their $\epsilon$ dependence both for smaller values of $\epsilon$ and over a wider range of values of $\epsilon$ than in previous studies.

First, as shown in Fig. 3, we find that the results for $\sqrt{\left\langle v^{2}\right\rangle}$ converge for $\epsilon \leqq 0.1$, where we have $\sqrt{\left\langle v^{2}\right\rangle} \propto \epsilon^{3 / 4}$. These results indicate that if we wish to study the characteristic spatiotemporal chaos exhibited by Eq. (1), we must choose a value of $\epsilon$ no greater than 0.1 . Then, as seen in Fig. 4, we find $\Delta q \propto \epsilon^{1 / 2}$, where $\Delta q$ is defined as the width at half maxi- 


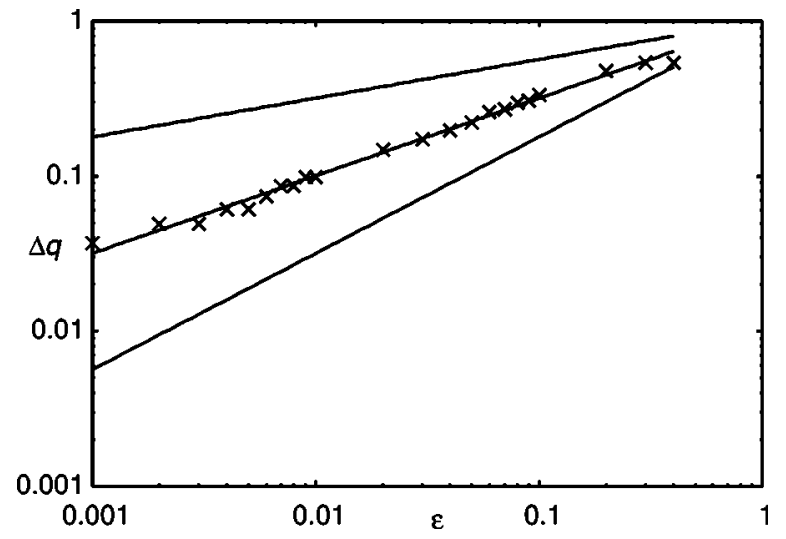

FIG. 4. $\epsilon$ dependence of $\Delta q$. The three lines, included for reference, have slopes of $1 / 4,2 / 4$, and $3 / 4$.

mum for the peak centered at $q \simeq 1$ of the spatial power density spectrum. This is evidence that the characteristic spatial scale of turbulent fluctuations is $\epsilon^{-0.5}$. Finally, we present in Figs. 5 and 6 the $\epsilon$ dependence of the spatial power densities $S\left(q_{0}\right) / L$ and $S\left(q_{c}\right) / L$ for the two characteristic modes $q_{0}$ and $q_{c}$. These are the wave numbers nearest to $q=0$ and $q=1$ (explicitly, $q_{0}=1 \times 2 \pi / L$ and $q_{c}=81 \times 2 \pi / L$ ) that can be realized in our system of size $L=2^{9}$ with periodic boundary conditions. From the figures it is seen that $S\left(q_{0}\right) \propto \epsilon^{3 / 2}$ and $S\left(q_{c}\right) \propto \epsilon^{1}$. The former relation cannot be seen as clearly as the latter, because, for larger wavelength modes, fewer wavelengths are contained in the finite-size space, and therefore there is a larger statistical error in the result, which is obtained by integrating Eq. (1) over a finite interval of time. Here, substituting $v=\epsilon^{\alpha} A(X, T) e^{i x}+$ c.c. $+\epsilon^{\beta} f(X, T)$ into the Wiener-Khintchine relation,

$$
S(q)=L \int_{0}^{L}\langle v(x) v(0)\rangle e^{-i q x} d x
$$

we obtain

$$
S(1)=L \int_{0}^{L} \epsilon^{2 \alpha}\langle A(X) \bar{A}(0)\rangle d x,
$$

where we have used $\langle A A\rangle=\langle\bar{A} \bar{A}\rangle=\langle A f\rangle=\langle\bar{A} f\rangle=0$. Now, because the characteristic spatial scale of turbulent fluctuations

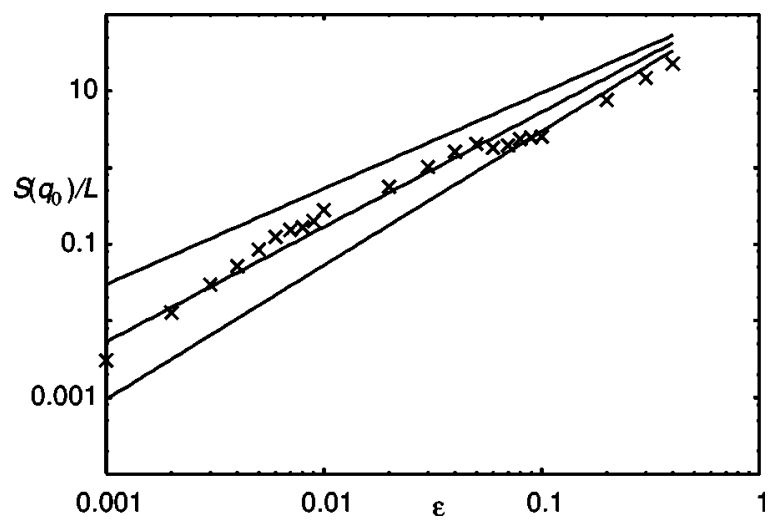

FIG. 5. $\epsilon$ dependence of $S\left(q_{0} \simeq 0\right)$. The three lines, included for reference, have slopes of $5 / 4,6 / 4$, and $7 / 4$.

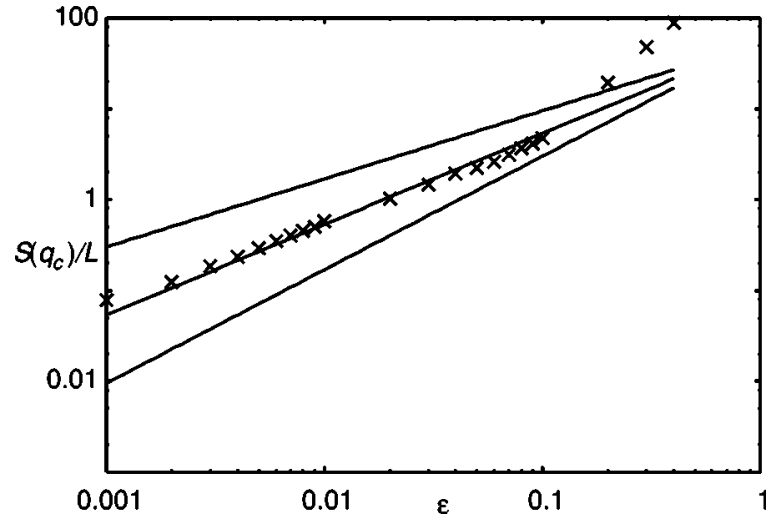

FIG. 6. $\epsilon$ dependence of $S\left(q_{c} \simeq 1\right)$. The three lines, included for reference, have slopes of $3 / 4,4 / 4$, and $5 / 4$.

is $\epsilon^{-1 / 2}$, as shown in Fig. 4, we can reasonably assume $\langle A(X) \bar{A}(0)\rangle=\exp \left[-\epsilon^{1 / 2} x\right]$. Then, substituting this into the integrand of Eq. (4), we obtain

$$
S(1)=L \epsilon^{2 \alpha-1 / 2} \text {. }
$$

(Here, we have used $L \epsilon^{1 / 2} \gg 1$. This is reasonable because we consider the sufficiently large system [19].) Comparing this equation and the result displayed in Fig. 6, we find $\alpha=3 / 4$, which is consistent with the result found for the exponent of $\sqrt{\left\langle v^{2}\right\rangle}$ obtained from Fig. 3. Similarly, we find

$$
S(0)=L \epsilon^{2 \beta-1 / 2},
$$

with $\beta=1[20]$. Thus, we arrive at the following conclusions. Our results confirm the validity of the form given in Eq. (2). Further, they indicate that the result for the exponent of $\sqrt{\left\langle v^{2}\right\rangle}$ given in Ref. [17] is erroneous because the value had not yet converged, as conjectured above. Equations (5) and (6) imply that the amplitudes of the Fourier modes with wave numbers $2 \pi / L(\simeq 0)$ and 1 defined in Ref. [9] are $2 \pi \epsilon^{1 / 4} / \sqrt{L}$ and $2 \pi \epsilon^{1 / 2} / \sqrt{L}$, respectively. Thus, our results indicate that the values of the quantity $\operatorname{Re} U / \sqrt{\epsilon}$ in Figs. 3 and 4 in Ref. [9] are of order 1 and 0.1, respectively, because the parameter values used there are $\epsilon=0.0001$ and $L=2 \pi / p$, where $p=3.125 \times 10^{-3}$. In fact, the figures in Ref. [9] support this argument. We believe that the reason why Matthews et al. reported that the form Eq. (2) is inconsistent with the numerical results in Ref. [9] is that they missed the $-1 / 2$ appearing in the exponents of Eqs. (5) and (6), which is due to the spatial correlation of turbulent fluctuations. At the end of this paragraph, although we consider the sufficiently large system in this paper, it is worthwhile mentioning that in numerical simulations one has to choose the system size $L$ in such a way that $L \epsilon^{1 / 2} \gg 1$, otherwise the $-1 / 2$ in the exponents of Eqs. (5) and (6) disappears. This implies that $\epsilon$ has also a lower bound $\left(\simeq L^{-2}\right)$ for simulations at fixed $L$ in order to observe the characteristic Nikolaevskii chaos.

In summary, we have found that the spatial power spectrum of Eq. (1) in wave-number space is an extensive quantity. The spectrum for $\epsilon \geqslant O(0.1)$ is qualitatively indistinguishable from that of the Kuramoto-Sivashinsky equation. We obtained the critical exponents of the turbulent fluctua- 
tions for Eq. (1), and we found that these exponents converge for $\epsilon \leqq O(0.1)$. Beyond such a value, because the unstable and weakly stable modes of this equation are not well separated, it exhibits spatiotemporal chaos of the KS type, not the Nikolaevskii type. Therefore, we conclude that the works presented in Refs. $[12,14,17]$, where values of $\epsilon$ greater than 0.1 were used, in fact studied KS-type chaos. The numerical results obtained in this paper are consistent with those given in Ref. [9] and with the amplitude equations appearing in Ref. [15]. Toh reported that a pulse-distributed model reproduces the spatial spectrum of the KS equation [18]. We believe that that model is applicable also to Eq. (1), for which pulses become more regularly distributed as $\epsilon$ decreases. Such studies might make it possible to estimate that the critical exponents converge for $\epsilon \lesssim O(0.1)$. The spectrum of the $\mathrm{KS}$ equation possesses a wavy structure for large wave numbers. We believe that for the spectrum of Eq. (1), the peaks of this wavy structure, which appear at $q=1,2,3, \ldots$, become increasingly sharp as $\epsilon$ decreases.

D. T. is very grateful to H. Fujisaka for useful discussions and gratefully acknowledges financial support by the Japan Society for the Promotion of Science (JSPS).
[1] A. M. Turing, Philos. Trans. R. Soc. London, Ser. B 237, 37 (1952).

[2] V. Castets, E. Dulos, J. Boissonade, and P. De Kepper, Phys. Rev. Lett. 64, 2953 (1990).

[3] Q. Ouyang and H. L. Swinney, Chaos 1, 411 (1991).

[4] See, e.g., N. Suzuki, M. Hirata, and S. Kondo, Proc. Natl. Acad. Sci. U.S.A. 100, 9680 (2003).

[5] D. Tanaka and Y. Kuramoto, Phys. Rev. E 68, 026219 (2003).

[6] D. Tanaka, Phys. Rev. E 70, 015202(R) (2004).

[7] V. N. Nikolaevskii, in Recent Advances in Engineering Science, edited by S. L. Koh and C. G. Speciale, Lecture Notes in Engineering Vol. 39 (Springer, Berlin, 1989), p. 210.

[8] M. I. Tribelsky and M. G. Velarde, Phys. Rev. E 54, 4973 (1996).

[9] M. I. Tribelsky and K. Tsuboi, Phys. Rev. Lett. 76, 1631 (1996).

[10] S. Kai, K. Hayashi, and Y. Hidaka, J. Phys. Chem. 100, 19007 (1996).

[11] H.-W. Xi, X.-J. Li, and J. D. Gunton, Phys. Rev. Lett. 78, 1046 (1997).
[12] H.-W. Xi, R. Toral, J. D. Gunton, and M. I. Tribelsky, Phys. Rev. E 62, R17 (2000).

[13] Y. Kuramoto, Chemical Oscillation, Waves, and Turbulence (Springer, New York, 1984); Chemical Oscillation, Waves, and Turbulence (Dover, New York, 2003).

[14] R. Toral, G. Xiong, J. D. Gunton, and H. Xi, J. Phys. A 36, 1323 (2003).

[15] P. C. Matthews and S. M. Cox, Phys. Rev. E 62, R1473 (2000).

[16] H. Fujisaka, T. Honkawa, and T. Yamada, Prog. Theor. Phys. 109, 911 (2003).

[17] I. L. Kliakhandler and B. A. Malomed, Phys. Lett. A 231, 191 (1997).

[18] S. Toh, J. Phys. Soc. Jpn. 56, 949 (1987).

[19] Alternatively, if $L \epsilon^{1 / 2} \ll 1$, we obtain $S(1)=L^{2} \epsilon^{2 \alpha}$. Using $L \epsilon^{1 / 2} \sim 1$, we can estimate the boundary between the two regimes that $S(q)$ is an extensive quantity, $S(q) \propto L$, or not, $S(q) \propto L^{2}$.

[20] If $L \epsilon^{1 / 2} \ll 1$, we obtain $S(0)=L^{2} \epsilon^{2 \beta}$. 